\title{
User interfaces applied to teleoperate mobile robots with keyboard command, PS3 controller and mobile phone
}

\author{
Nancy Velasco E, Darío José Mendoza Chipantasi, Antonio Barrientos Cruz. \\ Centro de Automática y Robótica \\ Universidad Politécnica de Madrid \\ Madrid, España \\ \{ndr.velasco, dario.mendoza.chipantasi\}@alumnos.upm.es, antonio.barrientos@upm.es
}

\begin{abstract}
User interfaces are part of our daily life, it is usual that people are connected to the digital world through applications in a computer, smart phone or tablet. At present time these devices offer a fluency feeling in real time communications, this is the principal argument to use them in this project. We present the design of three types of user interfaces employing Matlab and Android, which constitute a bridge between the movements that the person desires and mobile robot with Bluetooth connectivity. For this objective we control robots by the inclination of phone without physical buttons, also for many people who like the videogames is possible to control it with a traditional controller and finally if not available any of the above, the robot can be controlled through a common keyboard.. In addition this paper show the design and control of two cheap mobile robots for the user interface demonstration which contain an Arduino like a main processing board and the case created with $3 d$ printer.
\end{abstract}

Keywords - mobile robots, teleoperate, multimedia interfaces, PS3 controller.

\section{INTRODUCTION}

Nowadays user interfaces development are becoming increasingly crucial. One of the most significant types of development is based in Android, the operating system that powers millions smartphones and tablets, fast and smooth with slick graphics [1].

The future is wireless and Bluetooth technology [2] is a favorite in the world of electronics enthusiasts where the data link "no physical connection" must be robust, reliable and secure. Moreover the operative system Android [3] is the most famous in the world [4]. Furthermore, in recent years research on mobile robots is gaining followers [5][6].

An action field of mobile robots is the teleoperation [7], teleoperation allows an operator in a specific place to execute a task on another place, possibly separated by large distances [8][9]. On other hand, game controllers are used for different objectives like [10][11], one of them is the PlayStation controller that contains enough buttons to program robot functions like the velocity, steering and connectivity.

In this paper we combine the potential of android, ease of Bluetooth wireless connection, focusing on design a small cheap mobile robot [12] teleoperated via a smartphone or pc.

The robot has an Arduino control board, a Bluetooth module for wireless communication, infrared or ultrasonic sensors (depending on the model) to avoid collisions.

The control allows to link the robot with the device operated by the user with different speeds and automatic avoidance of obstacles, the control stops the movements of robot with the exception of reversing.
The rest of this paper is organized as follows. Section II: gives a simple summary about the user interfaces developed for control the mobile robots using keyboard, ps3 controller and mobile phone. Section III presents the design of robots, information about theirs parts and performance, as well as design drawings with their respective views. The experiments are given in Section IV. Finally, Section V is the conclusion.

\section{USER INTERFACES}

\section{A. The heart of user interfaces}

The user interfaces are the skin of applications they represent the link between the codes that be sending via Bluetooth and the friendly user interfaces. Always the best program or application need be simple and intuitive; for these reasons we designed three different interfaces, but the heart of communication of them are the same.

These robots used Bluetooth technology that is mainly based on connect two devices together with the same settings. A brief explanation about the parameters for serial communication is listed below:

$$
\begin{aligned}
& \text { Baud Rate = 9600; } \\
& \text { Byte Size = 8; } \\
& \text { Stop Bits = ONE STOP BIT; } \\
& \text { Parity = NON PARITY; }
\end{aligned}
$$

Once configured these parameters on both mobile robot and control device, the robots are ready to begin the transmission of data. We devise a common communication for send the same data although we change the user interface, 
The possible movements are forward, backward, left and right, the speeds that can be chosen are: fast, normal and slow, we contemplated that if the user does not press the scroll keys, the robot will stop.

\section{B. PS3 Controller Interface}

The DUALSHOCK 3 wireless controller for the PlayStation 3 system provides the most intuitive game play experience with pressure sensors in each action button and the inclusion of the highly sensitive SIXAXIS technology motion detection. Each hit, crash and explosion is more realistic when the user feels the rumble right in the palm of your hand. Use Bluetooth wireless technology for gaming [13].

An user interface Fig. 1 was created in Matlab to control robots using the PS3 controller, this program allows you perform movements such as forward, backward, left and right, as they have pressed the corresponding keys on the controller, plus you can control the speed of moving in three distinct

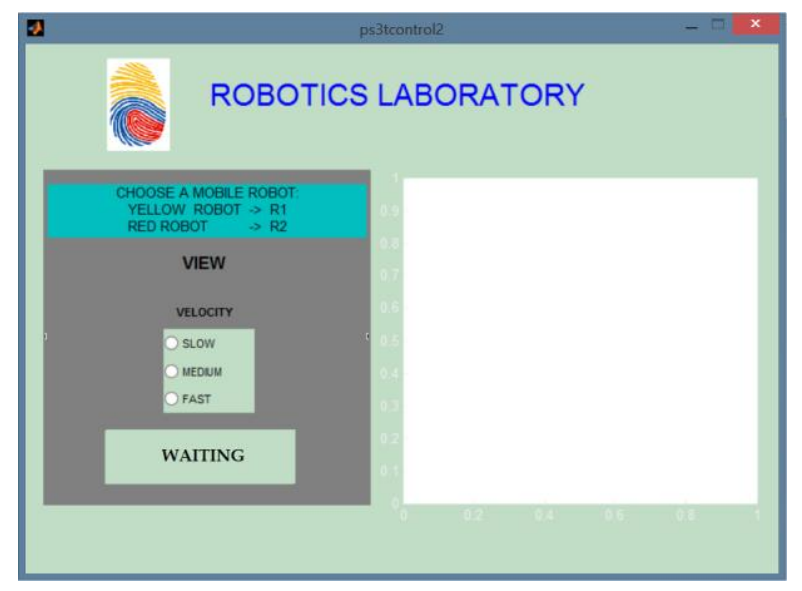

Fig. 1. Interface for $\mathrm{ps} 3$ controller

stages: the speeds that can be selected are low, medium, high.

First we set the control to work in Matlab with mfiles of JOYMEX [14]. These platforms lead the development of this work;

We use mfiles of JOYMEX because it interprets the signals sent by each button of ps3 controller, for this way we can change or finish the control. Although we do not use in this project, ps3 controller has there buttons that give pressure information on them and even gives the rotation of the ps3 controller, for our project we focus on recognizing the data from the buttons are explained below;

\section{i. Selecting the robot}

- Yellow mobile robot- must press the "R1" button.

- Red mobile robot - must press the "R2" button.

ii. After selecting the robot with which we interact, we must expect the GUIDE notify us that found within the range of the robot and start connecting calls via Bluetooth, for pairing must press the "L1" button and wait the next step.

iii. Selecting the moving speed of the robot:

- Slow - button "triangle".

- Medium - button "circle".

- Fast - button "X".

iv. Controlling the robot with the arrow keys on the remote:

- Forward

- Right

- Backward

- Left

v. terminate the application press the "START" RC button

\section{Keyboard Interface}

In the application Fig. 2 the mobile robot is selected (yellow or red) and also the speed of movement, when you click on the start button, the application sets the Bluetooth communication, the status indicator will change from offline to online. The control keys of mobile robot are:

- w: forward

-d: right

- s: backward

- a: left

The application is terminated with finish button.

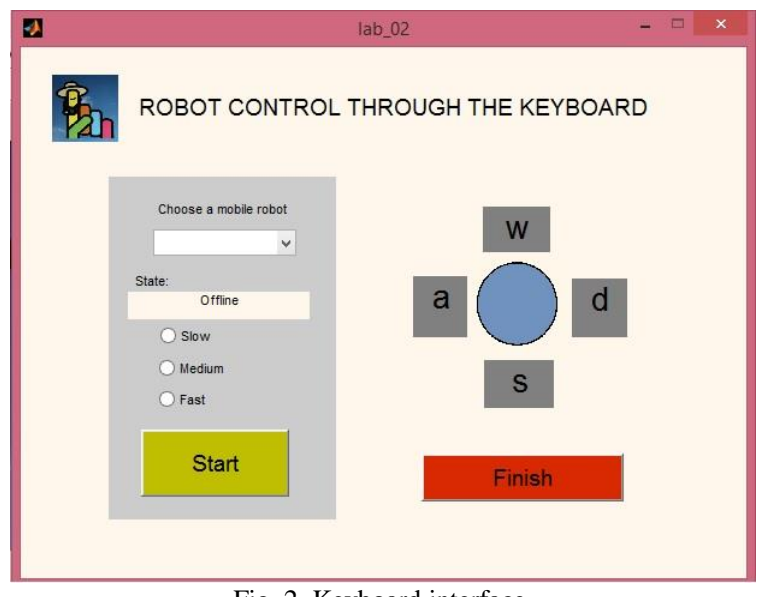

Fig. 2. Keyboard interface.

\section{Phone Interface}

The application Fig. 3 has the following elements:

- Power button, when pressed and select the device to be connected, the phone can be used as a remote control.

- A connection status indicator, it is connected (green) and offline (red).

- Four indicators are showing the move instruction, (left, right, up, back) and speed (yellow = slow, 
orange $=$ Mede, and red $=$ fast). The control is sensitive to the phone inclination, if the phone inclination increase, the velocity will bigger, but when the phone is centered the indicators are gray and the robot is stopped.

- Shutdown button to terminate the application.

The application has designed similar to following images:

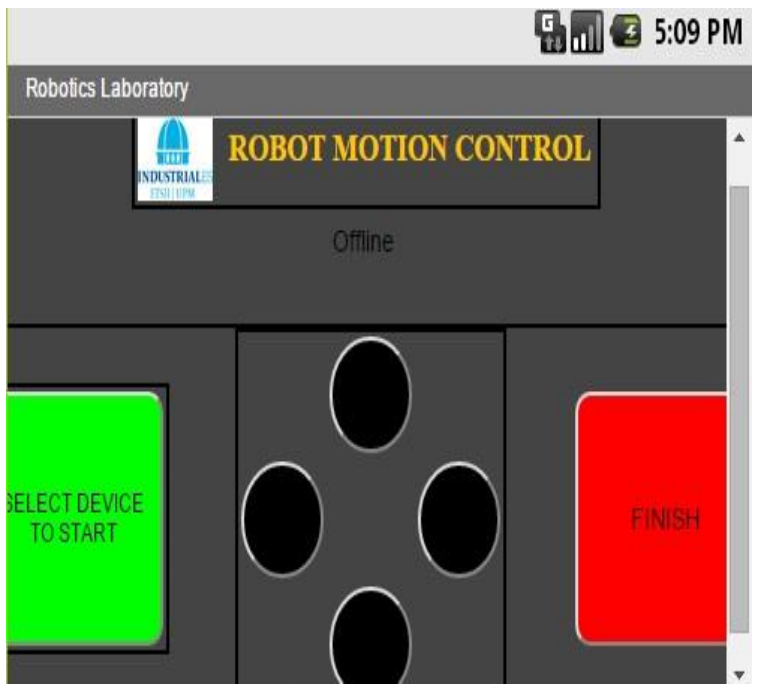

Fig. 3. Phone Interface

\section{MOBILE RoBOTS DESIGN}

We design and use two robots for the user interface demonstration.

The idea was create a cheap robot mobile, we decided to make our own designs and printed them, for this we use Inventor software, the design has complex forms not only because they look better aesthetically, but also for its functionality, we place two sensors at 45 degrees with respect at frontal sensor to avoid collisions, in the lower front the robot has an proximity infrared sensor to prevent the robot falling off the table or even for use it as a line follower.

Robots assembled and painted are in Fig. 4.

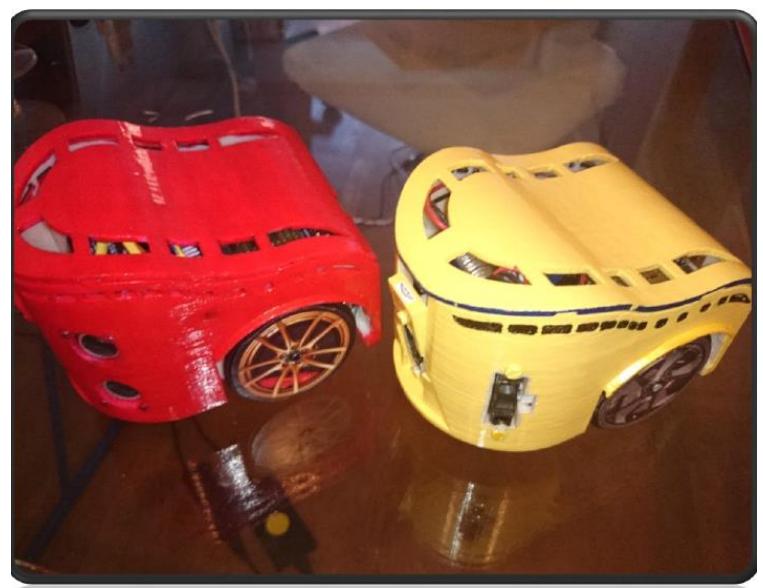

Fig. 4. Mobile Robots Design

\section{A. General Recognition of the Robots}

The views of mobile robots are in Fig. 5. Parts shown include:

1. On-off switch Battery.

2. Infrared Proximity Sensors

3. Ultrasonic Sensors

4. Floor sensor

5. Holes for jockey wheel

6. Motors

7. Lithium Polymer (LIPO).

8. Area circuitry.

Mobile robots have Arduino boards that are open platforms for prototyping with flexible software, inexpensive and easy to use.

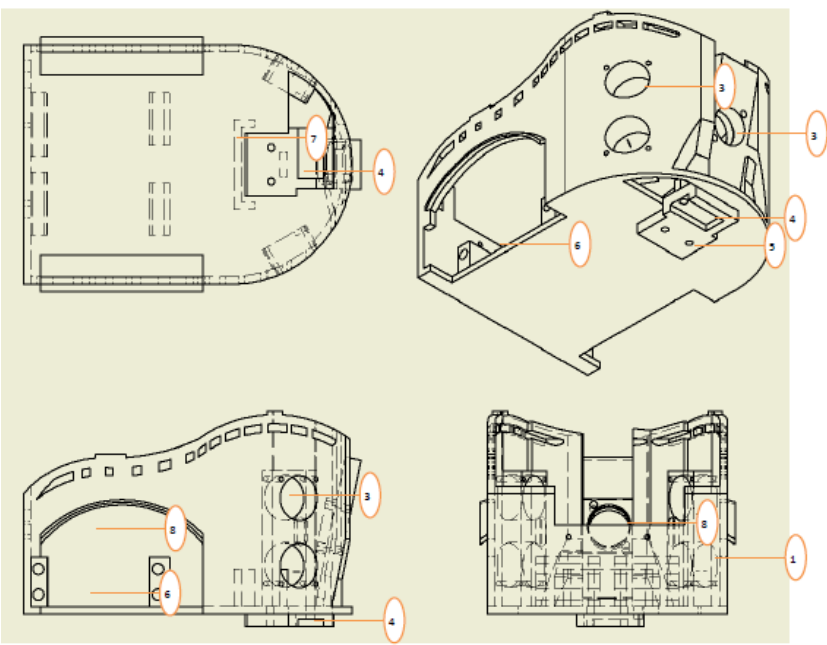

(a)
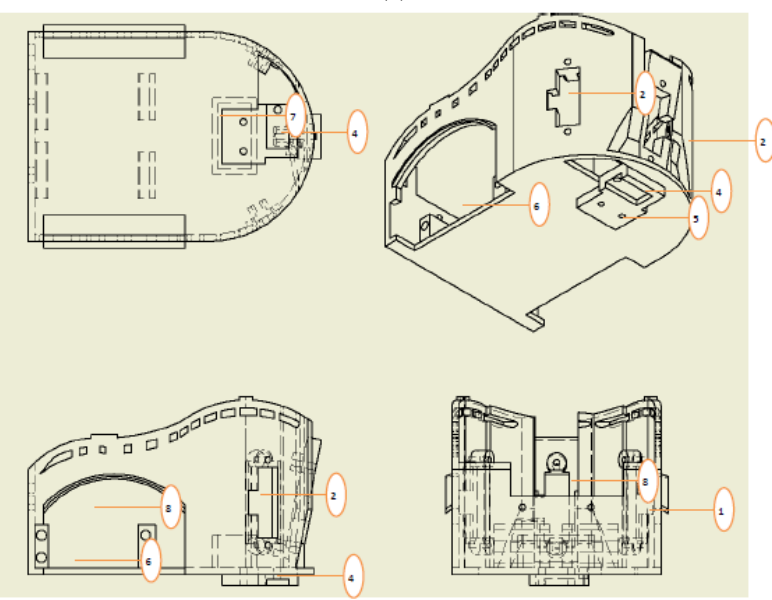

(b)

Fig.5. Views of mobile robot. (a) Robot with Ultrasonic Sensors and (b) Robot Infrared Proximity Sensors 


\section{EXPERIMENTS}

In our work, we designed, printed and developed two mobile robots that are controlled independently with multiple devices. We try to prove that it is easy to merge several technologies to make interesting projects with inexpensive cost and with customizable user interfaces, for us the most innovative controller was the smartphone because it is able to control robots by the inclination without physical buttons, but for many people who like the videogames is also possible to control it with a traditional controller and finally if not available any of the above, the robot can be controlled through a common keyboard.

Experiments with the ps3 controller allowed to conclude that the interface was adequate for handling the mobile robot, since many users are familiarized to the control because it is a command for video games. Fig. 6 shows the application that run and the user with ps3 controller.

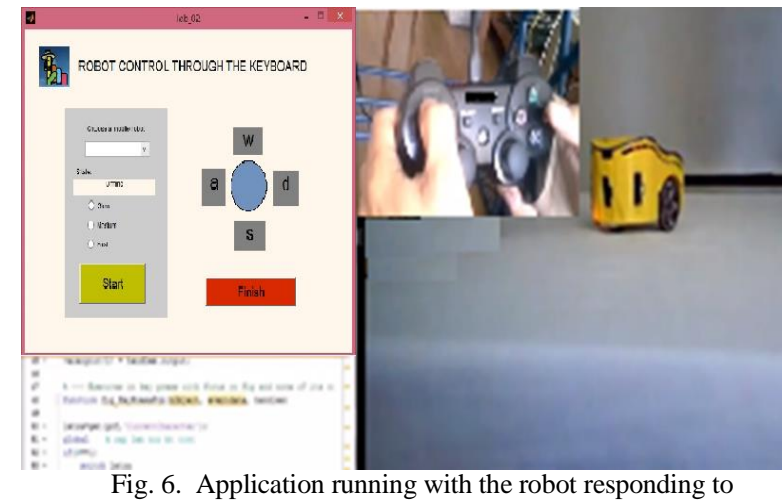
command

Experiments with the keyboard were efficient, each person that operate a computer has used a keyboard, so the interface is simple to use. The Fig. 7. Shows the application and the robot.

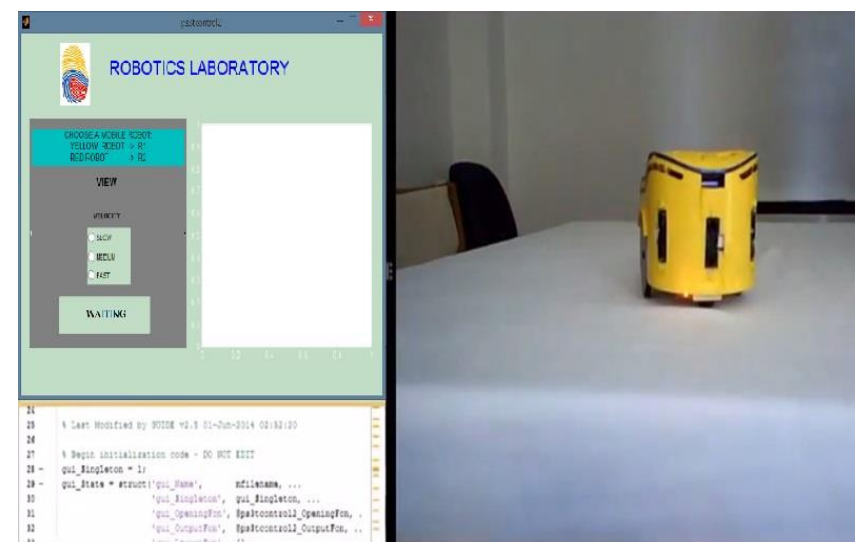

Fig. 7. Keyboard Application and the robots responding to command.

Experiments with the phone were the most intuitive control using the accelerometer [15] as sensor, thereby moving the phone in one direction and with a certain inclination user can generate a slow or fast motion or the detention of the robot

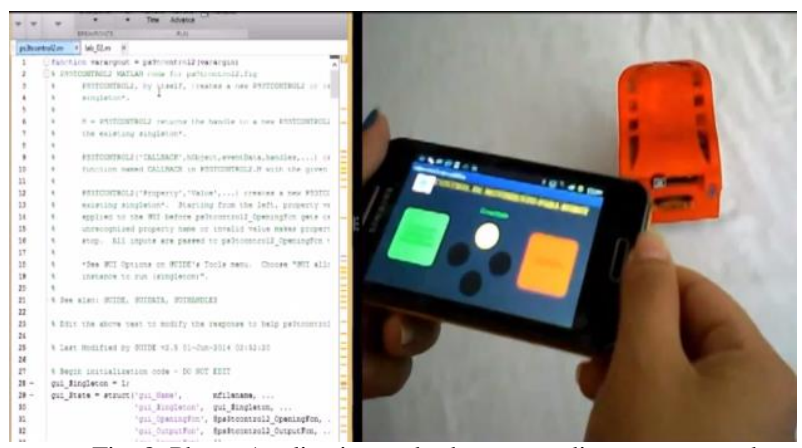

Fig. 8. Phone Application and robot responding to command.

when is centered. Fig. 8. Shows this action.

\section{ACKNOWLEDGMENT}

Extensive gratitude to Professor Antonio Barrientos for having proposed the development of this challenge. Nancy Velasco and Darío Mendoza are supported by the Secretaría Nacional de Educación Superior, Ciencia, Tecnología e Innovación SENESCYT (Quito, Ecuador) under Convocatoria Abierta 2013 Scholarship Program.

\section{REFERENCES}

[1] Lu Ying; Tang Xiao-jun; Liu Na; Mao Yu-Yue; Li Ming-Xia; Xiao Peng; Wang Hai-Wen, "Application and Research of Mobile Terminal with Android in the Equipment Monitoring System," Parallel Architectures, Algorithms and Programming (PAAP), 2014 Sixth International Symposium on , vol., no., pp.293,296, 13-15 July 2014

[2] Davies, A.C., "An overview of Bluetooth Wireless TechnologyTM and some competing LAN standards," Circuits and Systems for Communications, 2002. Proceedings. ICCSC '02. 1st IEEE International Conference on , vol., no., pp.206,211, 2002.

[3] Djajadi, A.; Putra, R.J., "Inter-cars safety communication system based on Android smartphone," Open Systems (ICOS), 2014 IEEE Conference on , vol., no., pp.12,17, 26-28 Oct. 2014.

[4] https://developer.android.com/about/index.html

[5] Ghayas, S.; Sulairnan, S.; Jaafar, J.; Mahammad, S., "Motivational scaffolding: Tackling the challenges of using mobile applications," User Science and Engineering (i-USEr), 2014 3rd International Conference on , vol., no., pp.48,51, 2-5 Sept. 2014.

[6] Vithani, T.; Kumar, A., "Presentation 5. A comprehensive mobile application development and testing lifecycle," IT Professional Conference (IT Pro), 2014 , vol., no., pp.1,3, 22-22 May 2014.

[7] Jianping Cai; Jianzhong Wu; Minghui Wu; Meimei Huo, "A bluetooth toy car control realization by android equipment," Transportation, Mechanical, and Electrical Engineering (TMEE), 2011 International Conference on , vol., no., pp.2429,2432, 16-18 Dec. 2011

[8] C. Sayers, Remote Control Robotics, Springer-Verlag, 1998.

[9] Alencastre-Miranda, M.; Munoz-Gomez, L.; Rudomin, I., "Teleoperating robots in multiuser virtual environments," Computer 
ICIT 2015 The $7^{\text {th }}$ International Conference on Information Technology

doi:10.15849/icit.2015.0108 @ ICIT 2015 (http://icit.zuj.edu.jo/ICIT15)

Science, 2003. ENC 2003. Proceedings of the Fourth Mexican International Conference on, vol., no., pp.314,321, 8-12 Sept. 2003.

[10] Nagata, F.; Watanabe, K., "Teaching system for a polishing robot using a game joystick," SICE 2000. Proceedings of the 39th SICE Annual Conference. International Session Papers, vol., no., pp.179,184, 2000.

[11] Ching-Chang Wong; Wei-Wen Wang; Ya-Ling Lee; Cheng-Hui Wu, "Remote controlled game platform by USB joysticks," Mechatronics, 2005. ICM '05. IEEE International Conference on , vol., no., pp.136,139, 10-12 July 2005.

[12] A. Barrientos, Fundamentos de Robótica, 2nd ed. McGraw-Hill, 2007.

[13] http://us.playstation.com/ps3/accessories/dualshock-3-wirelesscontroller-ps3.html

[14] http://joymex.escabe.org/

[15] http://www.androidpit.es/funcionamiento-acelerometro-smartphones 